\title{
POSITIVE $P_{N}$ CLOSURES
}

\author{
CORY HAUCK AND RYAN G. MCCLARREN
}

\begin{abstract}
We introduce a modification to the standard spherical harmonic closure used with linear kinetic equations of particle transport. While the standard closure is known to produce negative particle concentrations, the modification corrects this defect by requiring that the ansatz used to the close the equations is itself a non-negative function. We impose this requirement via explicit constraints in a quadratic optimization problem.
\end{abstract}

Keywords: kinetic equations, transport equations, neutron transport, moment closures, optimization, quadratic programming, spherical harmonic expansion, $P_{N}$ equations

AMS Classification: 70F45, 82D75, 65M99, 90C20, 41A29

\section{INTRODUCTION}

Linear transport problems arise in many areas of computational physics. Classic examples include the migration of neutrons in a nuclear reactor [22] or in an oil well [35], radiative heat transfer [30,34], neutrinos in supernovae [29], and charged particle transport in semiconductors [23]. Although described by linear equations, transport problems are difficult from a computational perspective as a result of the rich phase space required to describe the kinetic distribution. In general the phase space for a linear transport problem is seven-dimensional with one temporal, three spatial, one energy, and two angular variables.

A well-known approach for discretizing the transport equation in angle is to use moment equations based on a truncated spherical harmonic expansion in the angular variable. The result is that, for an order $N$ expansion, a single linear equation in seven variables is replaced by a linear system of $(N+1)^{2}$ unknowns in five variables. ${ }^{1}$ The equations in this system are often referred to colloquially as the $P_{N}$ equations.

The $P_{N}$ equations possess several desireable properties. For instance, the $P_{N}$ equations are, like the transport equation, rotationally invariant. ${ }^{2}$ This is in contrast to a discrete ordinates $\left(S_{N}\right)$ treatment of the angular component, which is not rotationally invariant and, as a consequence, suffers from asymmetries in the solution known as ray effects [22,24]. Moreover, the $P_{N}$ equations converge formall converge in an $L^{2}$ setting to the solution of

Date: July 13, 2009.

Los Alamos Report LA-UR 09-03865.

${ }^{1}$ This is for three-dimensional geometries. In two-dimensional geometries, a single linear equation in six variables is replaced by a linear system of $\left(\begin{array}{c}N+2 \\ 2\end{array}\right)$ unknowns in five variables. In one-dimensional geometries, a single linear equation in four variables is replaced by a linear system of $N+1$ unknowns in three variables.

${ }^{2}$ The definition of rotational invariance will be made more precise in the next section. 
the transport equation as $N \rightarrow \infty$. (For a sampling of rigorours results, see $[7,17,37]$.) Nevertheless, like reduced models in many other applications that are derived from linear approximations, the $P_{N}$ equations tend to suffer from undesirable artifacts of the linear expansion that defines them. Chief among these artifacts is the existence of solutions that are inconsistent with a positive particle concentration. Indeed, in multi-dimensional geometries or curvilinear geometries, the particle concentration produced by the $P_{N}$ equations can be negative for any finite order of $N[5,28]$. Besides being a distasteful nonphysical artifact, these negative particle concentrations can cause difficulties for the simulation of coupled physics operators.

There are other drawbacks to the $P_{N}$ equations. For instance, they treat particles as a series of linear waves that travel at speeds slower than the true particle speed. The effects of this reduced propagation speed are most noticeable with low order expansions and low collision rates $[27,32]$. Another issue is that the steady-state equations are ill-posed. In particular, the steady-state operator has a non-trivial nullspace, so that the steady-state solution is not unique [22]. In addition, there is no general theory to prescribe boundary conditions for $P_{N}$, although some progress has been made [11,19-21,33]. A consequence of these problems is that spherical harmonic approximations are less popular in practice than other methods such as implicit Monte Carlo [8] and discrete ordinates discretizations [22].

The motivation for our current work is the onset of negative particle concentrations produced by the $P_{N}$ equations. To this end, we introduce a modification of the $P_{N}$ closure that enforces positivity ${ }^{3}$ of the ansatz which approximates the kinetic distribution and its angular average. We do so by first reformulating the $P_{N}$ closure as the solution to a quadratic optimization problem with equality constraints. We then enforce positivity of the ansatz via the addition of inequality constraints. In practice, the new optimization problem must be solved on a discrete set of points in the angular variable.

Solving this new optimization problem is more computationally expensive than using the standard $P_{N}$ closure. Moreover, solutions must be realized locally at each point in space, time, and energy. Thus, in most cases, the new closure is not practical for serial computations. However, two mitigating factors make the closure a reasonable alternative for large-scale simulation. First, reconstructions can be performed in parallel across the spatial grid, and for a first-order scheme, the amount of data that is transferred between local computational cells is the same as for the standard closure. Thus, the closure procedure falls squarely into the emerging paradigm of data-parallel computing, since the extra work needed to solve the optimization can be handled by local processing units. The second point to make is that, in regions where the $P_{N}$ approximation to the transport solution is positive, the modified closure reduces to the standard $P_{N}$ closure. Thus the modification is really only needed in regions with strong discontinuities, boundary layers, or strong transient solutions.

The remainder of the paper is organized as follows. In section 2, we briefly recall the $P_{N}$ equations for a system of mono-energetic particles. In section 3, we discuss the problem of negative scalar fluxes inherent to the multi-dimensional setting, but also present a detailed

${ }^{3}$ In the context of this paper, positive is synonymous with positive, as opposed to strictly positive. 
analysis in the one-dimensional setting. In section 4, we give a variational formulation for the standard $P_{N}$ closure and, based on this formulation, introduce the new optimization problem which enforces positivity of the kinetic density. We also give implementation details in one and two dimensions. In section 5 we present numerical results for two benchmark problems. Concluding remarks and discussion for further work are given in section 6 .

\section{The $P_{N}$ Equations}

The $P_{N}$ equations are used to approximate the evolution of a kinetic distribution $F$ that is a function of position $r=(x, y, z) \in \Gamma \subset \mathbb{R}^{d}$, angle $\Omega \subset \mathbb{S}^{2}$, and time $t \geq 0$. For any measurable set $\mathcal{D} \subset \Gamma \times \mathbb{S}^{2}$

$$
\int_{\mathcal{D}} F(r, \Omega, t) d \Omega d r
$$

gives the number of particles at time $t$ that are characterized by a state $(r, \Omega) \in \mathcal{D}$. We assume that $F$ is governed by a kinetic transport equation of the form

$$
\partial_{t} F+\Omega \cdot \nabla_{r} F+\sigma F=\frac{\sigma}{4 \pi}\langle F\rangle
$$

where angled brackets denote integration with respect to the Lebesgue measure $d \Omega$ on the unit sphere $\mathbb{S}^{2}$. Note that the description provided by $F$ and the form of the governing kinetic equations assumes that particles are mono-energetic with speed one (otherwise the time derivative in (2) is divided by the speed) and that particle interactions are restricted to isotropic scattering events characterized by the cross-section $\sigma=\sigma(r)$.

Derivation of any moment system begins with a the choice of a vector-valued function $\mathbf{p}: \mathbb{S}^{2} \rightarrow \mathbb{R}^{s}$, whose $s$ components are linearly independent functions of $\Omega$. Exact equations for the moments $\mathbf{u}(r, t):=\langle\mathbf{p} F(r, \Omega, t)\rangle$ are found by multiplying the transport equation by $\mathbf{p}$ and integrating over all angles. This gives the system

$$
\partial_{t} \mathbf{u}+\nabla_{r} \cdot\langle\Omega \mathbf{p} F\rangle+\sigma\langle\mathbf{p} F\rangle=\frac{\sigma}{4 \pi} \rho\langle\mathbf{p}\rangle,
$$

where $\rho=\langle F\rangle$ is the particle concentration. This system is closed by approximating

$$
F(r, \Omega, t) \simeq \mathcal{F}(\mathbf{u}(r, t), \mathbf{p}(\Omega)),
$$

in (3). At this level, the only requirement on the the ansatz $\mathcal{F}$ that satisfies the consistency relation

$$
\langle\mathbf{p} \mathcal{F}(\mathbf{u}, \mathbf{p})\rangle=\mathbf{u} .
$$

Standard $P_{N}$ equations are derived in this abstract framework by selecting spherical harmonics as the components of $\mathbf{p}$ and expressing the ansatz $\mathcal{F}$ as a linear expansion of these components:

$$
\mathcal{F}(\mathbf{u}(r, t), \Omega)=\mathbf{c}^{T} \mathbf{p}, \quad \mathbf{c}=\left\langle\mathbf{p p}^{T}\right\rangle^{-1} \mathbf{u}
$$


where the expansion coefficients in the vector $\mathbf{c}$ are determined by the consistency relation (5).The form of this reconstruction ensures that rotational invariance of the transport operator is maintained. ${ }^{4}$

\section{Negative Solutions}

Solutions of the transport equation (2) are known to be positive as long as the initial condition and cross-section are also positive [6]. Unfortunately, this fundamental property is not retained by the $P_{N}$ system. In this section, we discuss the onset of negative scalar concentrations to the $P_{N}$ solutions in multi-dimensional settings, and then provide a more detailed analsis for the one-dimensional problem.

3.1. Multi-D. The negative particle concentrations produced by the $P_{N}$ equations in multi-dimensional settings are a consequence of the moment-based reconstruction in (6), which is spectral in the angular variable. Thus no mechanism exists to ensure pointwise bounds when discontinuities in the kinetic density lead to oscillations in the reconstruction.

One common example of a discontinuity is a shadow: at the edge of the shadow the particle density suffers a jump. For instance, in radiative transfer applications a negative particle concentration can lead to negative material energy densities and temperatures [28]. Such a result is given in Figure 3.1, where a simplified 2D hohlraum problem [3] is solved with $P_{11}$. This problem has discontinuities (shadows) behind the block as radiation flows from the source on the left of the problem. The $P_{11}$ equations reconstruct the solution near this discontinuity with a negative particle concentration.

\footnotetext{
${ }^{4}$ It is straight-forward to show that the transport operator $\mathcal{T}: f \mapsto \Omega \cdot \nabla_{r} f+\sigma(f-\langle f\rangle)$ commutes with the rotation operator $\mathcal{R}: f(r, \Omega, t) \mapsto f\left(O^{T} r, O^{T} \Omega, t\right)$ for any orthogonal matrix $O$. The $P_{N}$ approximation maintains this property. In particular, if $\mathcal{P}$ is the project onto the expansion defined in (6), then the operator $\mathcal{P} \circ \mathcal{T}$ also commutes with $\mathcal{R}$.
} 


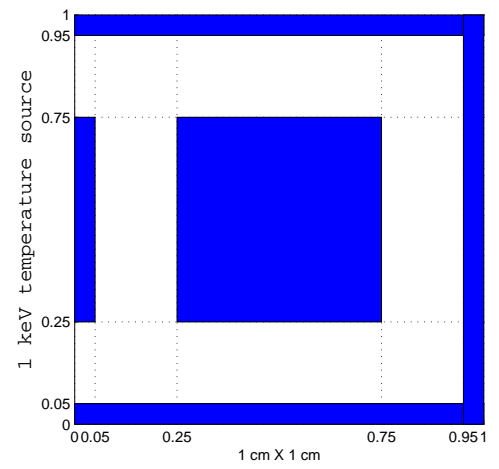

(a) Layout for the 2-D hohlraum problem: blue regions have $\sigma_{\mathrm{a}}=100$ and white regions have $\sigma_{\mathrm{a}}=0$.

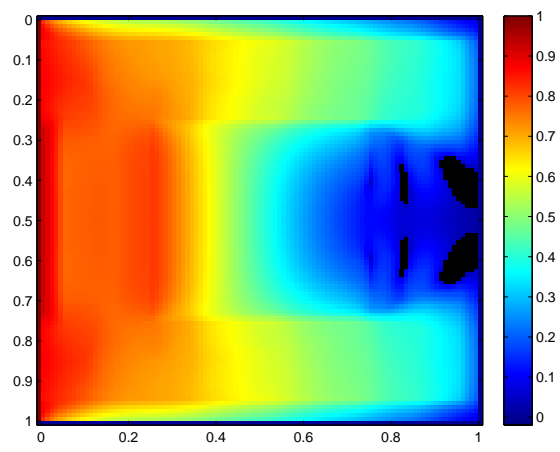

(b) Radiation temperature from a $P_{11}$ solution at $t=1$ nanosecond. Black denotes negative values

Figure 1. Example of negative particle concentrations in a $P_{N}$ solution for a radiative transfer problem.

The canonical benchmark problem which exemplifies negative particle concentrations for the $P_{N}$ equations is the so-called linesource problem $[5,10,27]$, in which particles are emitted into a weakly scattering medium by a pulsed, infinite line of particles. As a consequence of symmetry, the problem can be formulated in two dimensions with a source represented by a Dirac delta at the origin. Several profiles of the particle concentration for the linesource problem are given in Figure 2. These include a semi-analytic solution to the transport equation $[9,10,12]$, along with numerical approximations of $P_{1}$ and $P_{3}$ that are computed with a discontinuous Galerkin code $[14,25,26]$. The transport solution has a singularity at a distance $t$ from the origin which at $t=1.0$ requires that we cut the solution off at $\rho=1.0$. The inability of the $P_{1}$ and $P_{3}$ equations to preserve the nonnegative property of the transport solution at short times (Figures 2(a) - 2(c)) is generic. However, this defect subsides at later times (Figures 2(d) - 2(f)), as more and more collisions occur and the $P_{N}$ expansion becomes more faithful approximation of the exact transport solution. 


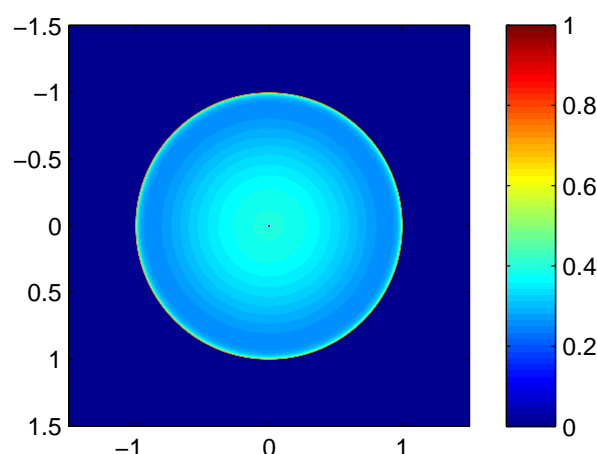

(a) Exact transport solution, $t=1.0$

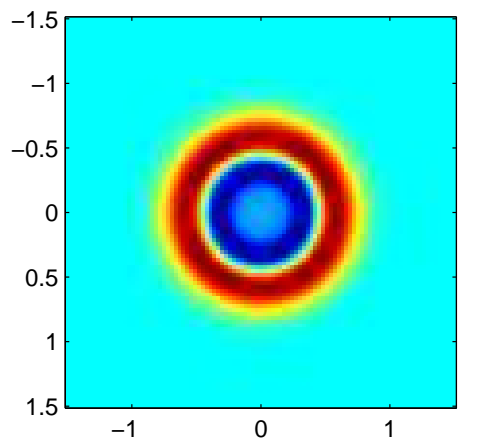

(c) $P_{1}, t=1.0$

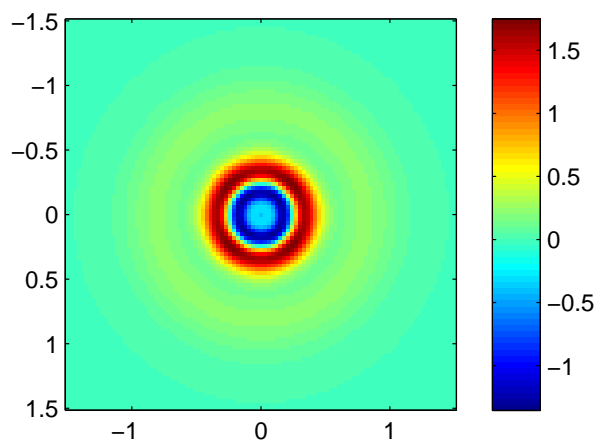

(e) $P_{3}, t=1.0$
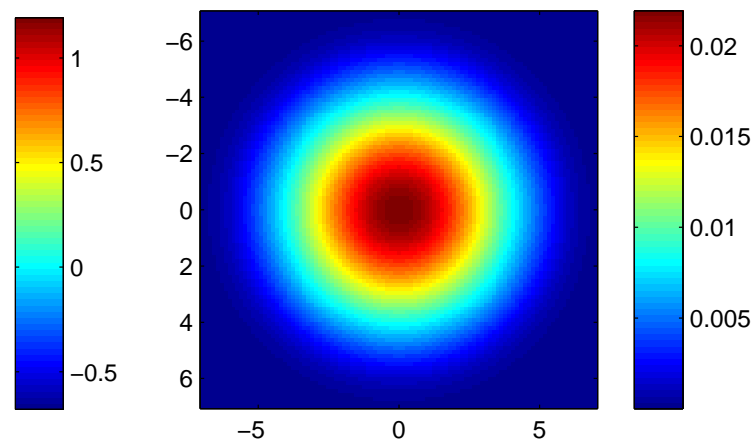

(d) $P_{1}, t=10.0$

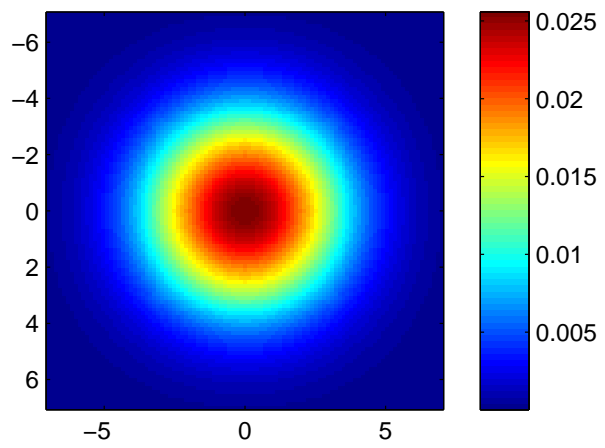

(f) $P_{3}, t=10.0$

Figure 2. The linesource in two dimensions. 
3.2. 1-D. Negative particle concentrations produced by the densities $P_{N}$ equations are an inherently multidimensional issue. Indeed, if initialized properly, solutions of the $P_{N}$ equations in one dimension give a positive particle concentration. Even so, valuable insight is gained by an analysis of the one-dimensional setting (i.e. for slab geometry), where $F$ is independent of the $x$ and $y$ variables. In such cases, the function

$$
\bar{F}(z, \mu, t):=\int_{0}^{2 \pi} F(r, \Omega, t) d \phi
$$

where $\mu$ is the $z$-component of $\Omega$ and $\phi \in[0,2 \pi)$ is the azimuthal angle, satisfies a reduced equation

$$
\partial_{t} \bar{F}+\mu \partial_{z} \bar{F}+\sigma \bar{F}=\frac{\sigma}{2} \rho
$$

and the spherical harmonic components of the vector $\mathbf{p}$ reduce to the the first $N+1$ Legendre polynomials in the variable $\mu$. The $P_{N}$ system derived from (8) takes the form

$$
\partial_{t} \mathbf{u}+A \partial_{z} \mathbf{u}=-\sigma Q \mathbf{u},
$$

where the flux matrix $A$ and the relaxation matrix $Q$ are given by

(10) $A_{\alpha \beta}=\frac{\alpha+1}{2 \alpha+1} \delta_{\alpha+1, \beta}+\frac{\alpha}{2 \alpha+1} \delta_{\alpha-1, \beta} \quad$ and $\quad Q_{\alpha \beta}=\delta_{\alpha \beta}\left(1-\delta_{\alpha, 0}\right), \quad \alpha, \beta=0, \ldots, N$.

The matrix $A$ is diagonalizable; its eigenvalues $\left\{\lambda_{0}, \ldots, \lambda_{N}\right\}$ are the zeros of the Legendre polynomial $p_{N+1}$ and form the $(N+1)$-point Gauss-Legendre quadrature set on the interval $[-1,1]$.

A particular choice of right and left eigenvectors diagonalizes the $P_{N}$ equations into an equivalent discrete ordinate form. To define this transformation, we let

$$
\Lambda=\operatorname{Diag}\left(\lambda_{0}, \ldots, \lambda_{N}\right), \quad P=\left[\mathbf{p}\left(\lambda_{0}\right), \ldots, \mathbf{p}\left(\lambda_{N}\right)\right], \quad \mathbf{w}=\left[w_{0}, \ldots, w_{N}\right],
$$

where $w_{\alpha}$ is the quadrature weight associated with $\lambda_{\alpha}$. Then the $(N+1) \times(N+1)$ matrices

$$
R=P \operatorname{Diag}(\mathbf{w}) \quad \text { and } \quad L=P^{T}\left\langle\mathbf{p p}^{T}\right\rangle^{-1}
$$

contain the right (column) and left (row) eigenvectors of $A$, so that $L R=R L=I$ and $A=L \Lambda R$. The vector $\mathbf{v}=\left(v_{0}, \ldots, v_{N}\right):=L \mathbf{u}=P^{T} \mathbf{c}$ satisfies the discrete ordinate equation

$$
\partial_{t} \mathbf{v}+\Lambda \partial_{z} \mathbf{v}+\sigma \mathbf{v}=\sigma\left(\frac{\boldsymbol{\rho}}{2}\right)
$$

where $\boldsymbol{\rho}=[\rho, \ldots, \rho]^{T}$. In particular, each $v_{\alpha}$ is a solution of (8) along the direction $\mu=\lambda_{\alpha}$, but with initial condition

$$
\mathcal{F}\left(z, \lambda_{\alpha}, 0\right)=\mathbf{c}^{T}(z, 0) \mathbf{p}\left(\lambda_{n}\right)=\left\langle\mathbf{p} \mathbf{p}^{T}\right\rangle^{-1}\left\langle\mathbf{p}^{T} F(z, \cdot, 0)\right\rangle \mathbf{p}\left(\lambda_{n}\right)
$$

If initially positive, then these solutions will remain so for all time [6]. Thus the particle concentration, which is given by $\rho=\mathbf{w}^{T} \mathbf{v}$, is also positive. 
In some cases, positivity of the $v_{\alpha}$ and of $\rho$ can be preserved by a numerical scheme. One such scheme is the following first order, semi-implicit, upwind (SIU) method for the spatial averages $\mathbf{v}_{i}^{n}$ on a uniform mesh:

$$
\mathbf{v}_{i}^{n+1}=\mathbf{v}_{i}^{n}-\frac{\Delta t}{\Delta z}\left[\Lambda^{+}\left(\mathbf{v}_{i}^{n}-\mathbf{v}_{i-1}^{n}\right)+\Lambda^{-}\left(\mathbf{v}_{i+1}^{n}-\mathbf{v}_{j}^{n}\right)\right]-\Delta t \sigma\left(\mathbf{v}_{i}^{n+1}-\boldsymbol{\rho}_{i}^{n+1}\right)
$$

or, in terms of the moments,

$$
\mathbf{u}_{i}^{n+1}=\mathbf{u}_{i}^{n}-\frac{\Delta t}{2 \Delta z}\left[A\left(\mathbf{u}_{i+1}^{n}-\mathbf{u}_{i-1}^{n}\right)-|A|\left(\mathbf{u}_{i+1}^{n}-2 \mathbf{u}_{j}^{n}+\mathbf{u}_{i-1}^{n}\right)\right]-\Delta t \sigma \mathbf{u}_{i}^{n+1}
$$

where $|A|=L|\Lambda| R$ and $\Lambda^{ \pm}=(\Lambda \pm|\Lambda|) / 2$.

Proposition 1. The numerical scheme (15)-(16) preserves the positivity of the components of $\mathbf{v}$ under the CFL condition $\Delta t<\Delta z$.

Proof. We write the scheme as

$$
\mathbf{v}_{i}^{n+1}=\gamma\left[C \mathbf{v}_{i}^{n}+C^{-} \mathbf{v}_{i-1}^{n}+C^{+} \mathbf{v}_{i+1}^{n}\right]+(1-\gamma) \boldsymbol{\rho}_{i}^{n+1},
$$

where the scalar

$$
\gamma:=\frac{1}{1+\sigma \Delta t}
$$

and the components of the diagonal matrices

$$
C:=\left(I-\frac{\Delta t}{\Delta z}|\Lambda|\right) \quad \text { and } \quad C^{ \pm}:=\frac{\Delta t}{\Delta z} \Lambda^{ \pm}
$$

are all positive under the assumed CFL condition. It remains only to show that $\rho_{i}^{n+1}$ is positive. However, this fact is trivial, since

$$
\rho_{i}^{n+1}=\mathbf{w}^{T}\left[C \mathbf{v}_{i}^{n}+C^{-} \mathbf{v}_{i-1}^{n}+C^{+} \mathbf{v}_{i+1}^{n}\right]
$$

and the components of $\mathbf{v}$ are positive.

Even though the reconstruction $\mathcal{F}$ is positive at the quadrature points $\lambda_{n}$, the same is not true for all values of $\mu$. Consider the following example.

Example 1. We apply SIU algorithm to a test problem with periodic boundary conditions. The initial condition for $\rho$ is a delta function at the origin (the so-called plane source); all other moments are initially zero, and $\sigma=1$.

In Figure 3, we plot results after time $t=1.0$. Figure 3(a) shows the profiles of the four discrete ordinate components of $\mathbf{v}$, which are all positive. Figure 3(b) shows the profile of $\rho$, which is just a weighted sum of these components and hence also positive. In Figure $3(\mathrm{c})$, we plot the minimum value of the reconstruction $\mathcal{F}$ at each point in space:

$$
\mathcal{F}^{*}:=\min _{\mu \in[-1,1]} \mathcal{F}(\mathbf{u}(z, 1), \mu) \text {. }
$$


It is clear that the angular reconstruction goes very negative near the peaks of the characterstic waves. Indeed, in the worst case, the ratio of $\left|\mathcal{F}^{*}\right|$ to the corresponding isotropic distribution is greater than one-that is,

$$
\max _{z \in[0,2]} \frac{\left|\mathcal{F}^{*}\right|}{\rho / 2}>1
$$

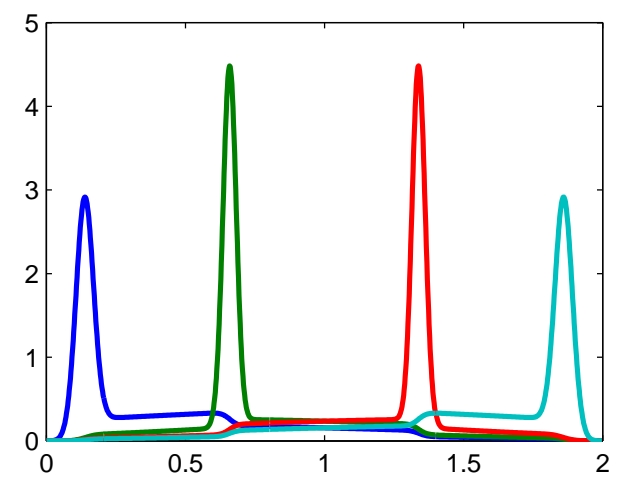

(a) Components of $\mathbf{v}$

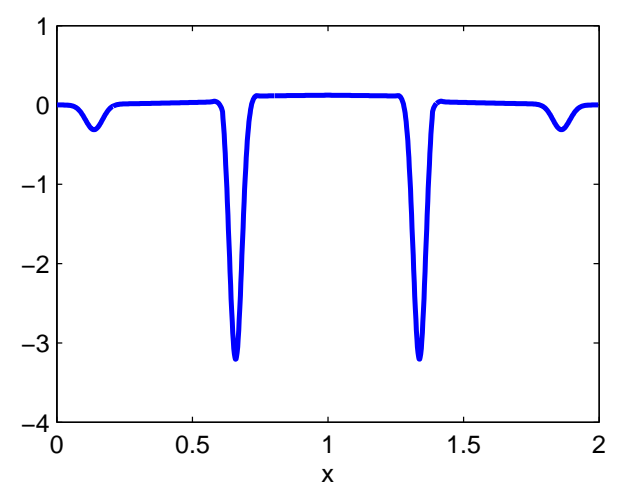

(c) $\min _{\mu \in[-1,1]} \mathcal{F}(\mathbf{u}(z, t=1), \mu)$

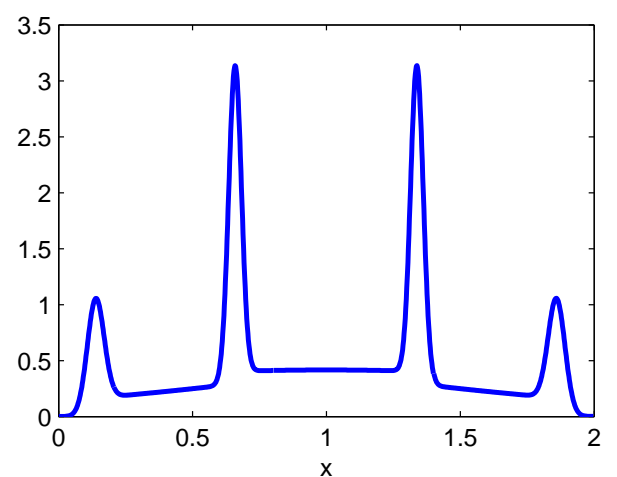

(b) Scalar flux, $\rho$

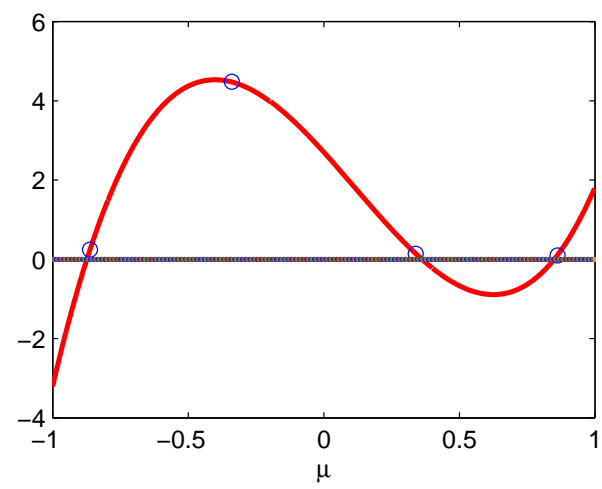

(d) $P_{3}$ reconstruction at $z=0.68$. Circles highlight quadrature points.

Figure 3. Results at time $t=1.0$ for the SIU scheme (15)-(16), applied to the $P_{3}$ equations and computed with 1000 spatial cells $(\Delta z=0.002)$.

In Figure 3(d), we give a plot of the reconstructed angular flux at the second peak, near $z=0.68$. The reconstruction is (just barely) positive at the quadrature points $\{ \pm 0.33998 \ldots, \pm 0.86114 \ldots\}$, but is negative near $\mu=-1.0$ and again around $\mu=0.5$. The 
fact that the $P_{N}$ reconstruction so strongly violates the positivity property of $F$ raises serious questions as to whether the $P_{N}$ equations can provide a faithful representation of the transport equation. It also motivates our work in the next section to introduce corrections which enforce positivity of the reconstruction in an explicit fashion.

\section{The Positive $P_{N}\left(P P_{N}\right)$ Closure}

In this section, we introduce a closure which guarantees positivity of the reconstruction $\mathcal{F}$ of the kinetic density and, consequently, the particle concentration $\rho$. The ansatz for this closure is the solution to a constrained, convex optimization problem that is motivated by a variational formulation of the standard $P_{N}$ closure.

4.1. The Optimization Problem. While the $P_{N}$ equations are usually derived by a straight-forward spherical harmonic expansion [34], the $P_{N}$ reconstruction itself can be formulated as the solution to the following optimization problem:

$$
\begin{aligned}
\text { minimize } & \frac{1}{2}\left\langle|f|^{2}\right\rangle \\
\text { subject to } & f \in L^{2}\left(\mathbb{S}^{2}\right), \quad\langle\mathbf{p} f\rangle=\langle\mathbf{u}\rangle,
\end{aligned}
$$

where the components of $\mathbf{p}$ are spherical harmonics. The Lagrangian $\mathcal{L}: L^{2}\left(\mathbb{S}^{2}\right) \times \mathbb{R}^{s} \times \mathbb{R}^{s} \rightarrow$ $\mathbb{R}$ corresponding to $(23)$ is

$$
\left.\mathcal{L}(f, \mathbf{d} ; \mathbf{u})=\frac{1}{2}\left\langle|f|^{2}\right\rangle-\mathbf{d}^{T}(\langle\mathbf{p} f\rangle-\langle\mathbf{u}\rangle)\right) .
$$

For a fixed value of $\mathbf{u}$, the solution of (23) corresponds to the saddle point of $\mathcal{L}$ at $(\mathcal{F}, \mathbf{c})$, where $\mathbf{c} \in \mathbb{R}^{s}$ solves the dual problem

$$
\text { minimize } \frac{1}{2}\left\langle\left|\mathbf{c}^{T} \mathbf{p}\right|^{2}\right\rangle-\mathbf{c}^{T} \mathbf{u}
$$

First order conditions at the saddle point of $\mathcal{L}$ imply that

$$
\frac{\delta \mathcal{L}}{\delta f}(\mathcal{F}, \mathbf{c} ; \mathbf{u})=0 \quad \text { and } \quad \frac{\partial L}{\partial \mathbf{d}}(\mathcal{F}, \mathbf{c} ; \mathbf{u})=0 .
$$

These two relations recover exactly the $P_{N}$ reconstruction given in (6).

To ensure positivity of $\mathcal{F}$, we modify the above prescription for the $P_{N}$ reconstruction by adding an inequality constraint as follows:

$$
\begin{aligned}
\text { minimize } & \frac{1}{2}\left\langle|f|^{2}\right\rangle \\
\text { subject to } & f \in L^{2}\left(\mathbb{S}^{2}\right),\langle\mathbf{p} f\rangle=\langle\mathbf{u}\rangle, f \geq 0 .
\end{aligned}
$$

Of course, enforcing such a constraint everywhere is generally not possible. In practice, we enforce the constraint on a finite quadrature set $\mathcal{Q}$ which is used to approximate the 
integrals in (27) and provides higher resolution of the angular variable of phase space than the components of $\mathbf{p}$. This leads to the following discrete version of (27):

$$
\begin{aligned}
\text { minimize } & \frac{1}{2} \sum_{\Omega_{k} \in \mathcal{Q}} w_{k}\left|f\left(\Omega_{k}\right)\right|^{2} \\
\text { subject to } & \sum_{\Omega_{k} \in \mathcal{Q}} w_{k} \mathbf{p}\left(\Omega_{k}\right) f\left(\Omega_{k}\right)=\langle\mathbf{u}\rangle, f\left(\Omega_{k}\right)>0 \forall \Omega_{k} \in \mathcal{Q},
\end{aligned}
$$

where $w_{k}$ is the weight corresponding to each quadrature point $\Omega_{k}$. A natural choice for $\mathcal{Q}$ is to use a standard discrete ordinate quadrature set $[15,22,31,36]$. Two examples of the optimization output are given in Figure 4.

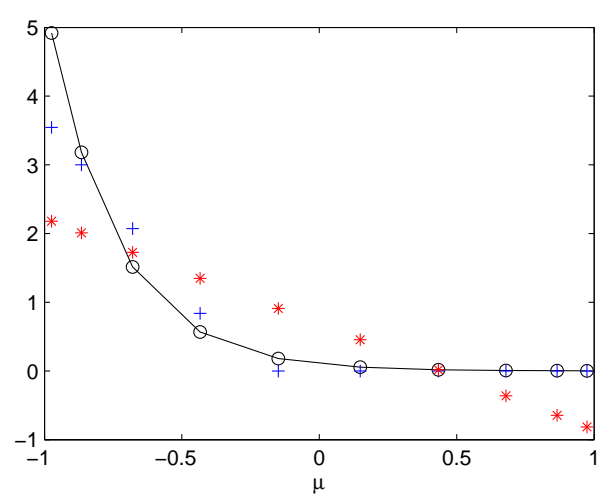

(a) $P_{1}$ vs $P P_{1}$

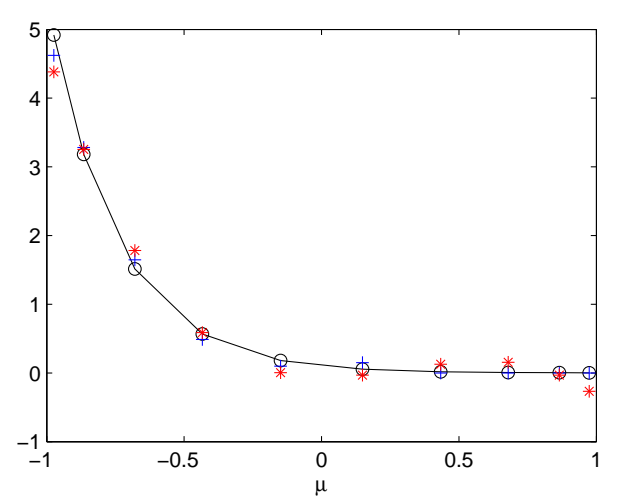

(b) $P_{3}$ vs $P_{3}$

FiguRE 4. Example optimization output. The underlying quadrature set $\mathcal{Q}$ is 10-point Gauss-Legendre. Circles are original function values on $\mathcal{Q}$ for $f(\mu)=0.1 \exp (4.0 \mu)$. Stars are the standard $P_{N}$ reconstruction. Pluses are the $P P_{N}$ reconstruction.

4.2. Implementation. Our implementation of the $P P_{N}$ closure follows in two basic steps. First, given moment data at time $t^{n}$, we solve the optimization problem in (27) to define an ansatz on each spatial cell. Each ansatz is then used to compute the numerical flux for an appropriate hyperbolic solver. The main challenge of this implementation is to ensure that the constraints in the optimization problem define a non-empty feasible set. In particular, the hyperbolic solver should not destroy feasibility at the next time level - that is, the values of the moments at $t^{n+1}$ must be consistent with a positive kinetic distribution. To ensure that this is indeed the case, we use a first-order, semi-implicit kinetic scheme. 
4.2.1. One Space Dimension. In one dimension, our semi-implicit algorithm is based on the one-dimensional transport equation (8). Let $\left\{z_{i}\right\}_{i \in \mathbb{Z}}$ be a set of uniformly spaced points that are centers of cells $C_{i}=\left[z_{i-1 / 2}, z_{i+1 / 2}\right]$ of width $\Delta z$ and let $\left\{t^{n}\right\}_{n \in \mathbb{Z}}$ be a set of uniformly spaced time intervals. Define

$$
\bar{F}_{i, k}^{n}=\frac{1}{\Delta z} \int_{C_{i}} \bar{F}\left(z, \mu_{k}, t^{n}\right) d z .
$$

A semi-implicit, upwind approximation of (8) is

$$
\frac{\bar{F}_{i, k}^{n+1}-\bar{F}_{i, k}^{n}}{\Delta t}+\max \left(\mu_{k}, 0\right) \frac{\bar{F}_{i, k}^{n}-\bar{F}_{i-1, k}^{n}}{\Delta z}+\min \left(\mu_{k}, 0\right) \frac{\bar{F}_{i+1, k}^{n}-\bar{F}_{i, k}^{n}}{\Delta z}+\sigma_{i} \bar{F}_{i, k}^{n+1}=\frac{\sigma_{i}}{2} \rho_{i}^{n+1},
$$

where $\sigma_{i}$ is an appropriately averaged value of $\sigma$ on $C_{i}$. (For the numerical results in the next section, $\sigma$ is a constant.) It is easy to show that the statement of Proposition 1 applies to this scheme, so that $\bar{F}_{i, k}^{n+1}$ is positive under a CFL condition $\Delta z \leq \Delta t$. To derive a scheme for moments, define the spatial averages

$$
\mathbf{u}_{i}^{n}=\frac{1}{\Delta z} \int_{C_{i}} \mathbf{u}\left(z, t^{n}\right) d z \equiv \frac{1}{\Delta z} \int_{C_{i}} \int_{-1}^{1} \mathbf{p}(\mu) \bar{F}\left(z, \mu, t^{n}\right) d \mu d z,
$$

and apply the quadrature rule associated with $\mathcal{Q}$ to (30), using the equality constraints in (28) to evaluate the moments and the source and loss terms. This gives the following kinetic scheme for the moments:

$$
\frac{\mathbf{u}_{i}^{n+1}-\mathbf{u}_{i}^{n}}{\Delta t}+\sum_{\mu_{k}>0} w_{k} \mu_{k} \mathbf{p}_{k} \frac{\bar{F}_{i, k}^{n}-\bar{F}_{i-1, k}^{n}}{\Delta z}+\sum_{\mu_{k}<0} w_{k} \mu_{k} \mathbf{p}_{k} \frac{\bar{F}_{i+1, k}^{n}-\bar{F}_{i, k}^{n}}{\Delta z}=-\sigma_{i} Q \mathbf{u}_{i}^{n+1}
$$

where $\mathbf{p}_{k}:=\mathbf{p}\left(\mu_{k}\right)$ and the components of $p$ are the Legendre polynomials [2] on the interval $[-1,1]$ :

$$
P_{l}(\mu)=\left\{\begin{array}{ll}
0 & , l=0 \\
\frac{1}{2^{l} l !} \frac{\partial^{l}}{\partial \mu^{l}}\left(\mu^{2}-1\right)^{l} & , l>0
\end{array} .\right.
$$

This kinetic scheme (32) is valid for any closure. To select a particular closure, one need only select an angular reconstruction for $\bar{F}$ in (29). For $P P_{N}$, this is done using the ansatz defined via the optimization problem in (28), where the quadrature set $Q$ is the $M$-point Gauss-Legendre quadrature set on $[-1,1]$ and $M \geq N+1$. We implement (32) in MATLAB, using the program quadprog to solve (28).

4.2.2. Two Space Dimensions. The implementation of the scheme in higher dimensions is straightforward. In two dimensions, we can write $F=F(x, y, \Omega, t)$. The transport equation for $F$ in this case is

$$
\partial_{t} F+\xi \partial_{x} F+\eta \partial_{y} F+\sigma F=\frac{\sigma}{4 \pi} \rho,
$$

where $\xi$ and $\eta$ are the $x$ and $y$ components of $\Omega$, respectively. 
Let $\left\{\left(x_{i}, y_{j}\right)\right\}_{(i, j) \in \mathbb{Z}^{2}}$ be a mesh of evenly spaced points that are centers of rectangular cells $C_{i j}=\left[x_{i-1 / 2}, x_{i+1 / 2}\right] \times\left[y_{j-1 / 2}, y_{j+1 / 2}\right]$ with dimensions $\Delta x$ and $\Delta y$. Let $\left\{t^{n}\right\}_{n \in \mathbb{Z}}$ be a set of evenly spaced time intervals. Define

$$
F_{i, j, k}^{n}:=\frac{1}{\Delta x \Delta y} \int_{C_{i}} F\left(x, y, \Omega_{k}, t^{n}\right) d x,
$$

where $\Omega_{k}$ is a element of the quadrature set $Q$. The semi-implicit upwind scheme for (34) is

$$
\begin{aligned}
\frac{F_{i, j, k}^{n+1}-F_{i, j, k}^{n}}{\Delta t} & +\max \left(\xi_{k}, 0\right) \frac{F_{i, j, k}^{n}-F_{i-1, j, k}^{n}}{\Delta x}+\min \left(\xi_{k}, 0\right) \frac{F_{i+1, j, k}^{n}-F_{i, j, k}^{n}}{\Delta x} \\
& +\max \left(\eta_{k}, 0\right) \frac{F_{i, j, k}^{n}-F_{i, j-1, k}^{n}}{\Delta y}+\min \left(\eta_{k}, 0\right) \frac{F_{i, j+1, k}^{n}-F_{i, j, k}^{n}}{\Delta y} \\
& =-\sigma_{i, j}\left(F_{i, j, k}^{n+1}-\frac{1}{4 \pi} \rho_{i j}^{n+1}\right),
\end{aligned}
$$

where $\sigma_{i, j}$ is an appropriately averaged value of $\sigma$ on $C_{i j}$. Integrating this discrete equation against $\mathbf{p}$ and applying the constraints in (28) gives

$$
\begin{aligned}
\frac{\mathbf{u}_{i, j}^{n+1}-\mathbf{u}_{i, j}^{n}}{\Delta t} & +\sum_{\xi_{k}>0, \eta_{k}} w_{k} \mathbf{p}_{k} \xi_{k} \frac{F_{i, j, k}^{n}-F_{i-1, j, k}^{n}}{\Delta x}+\sum_{\xi_{k}<0, \eta_{k}} w_{k} \mathbf{p}_{k} \xi_{k} \frac{F_{i+1, j, k}^{n}-F_{i, j, k}^{n}}{\Delta x} \\
& +\sum_{\xi_{k}, \eta_{k}>0} w_{k} \mathbf{p}_{k} \eta_{k} \frac{F_{i, j, k}^{n}-F_{i, j-1, k}^{n}}{\Delta y}+\sum_{\xi_{k}, \eta_{k}<0} w_{k} \mathbf{p}_{k} \eta_{k} \frac{F_{i, j+1, k}^{n}-F_{i, j, k}^{n}}{\Delta y}=-\sigma_{i, j} Q \mathbf{u}_{i, j}^{n+1},
\end{aligned}
$$

where $\mathbf{p}_{k}:=\mathbf{p}\left(\Omega_{k}\right)$. As in the one-dimensional case, the closure is implemented by finding an ansatz to approximate $F_{i, j, k}^{n}$. The two-dimensional algorithm is also implemented in MATLAB, using quadprog to solve the optimization problem (28).

In general, the spherical harmonics components of $\mathbf{p}$ take the form [2]

$$
Y_{l}^{m}(\theta, \phi)=\sqrt{\frac{2 l+1}{4 \pi} \frac{(l-m) !}{(l+m) !}} P_{l}^{m}(\cos \theta) e^{i m \phi} .
$$

Here, $\theta$ and $\phi$ are the polar and azimuthal angles, respectively, associated to $\Omega$, so that

$$
\Omega=(\xi, \eta, \mu)=(\sin \theta \cos \phi, \sin \theta \sin \phi, \cos \theta) .
$$

The degree $l$ and order $m$ are integers such that $0 \leq l \leq N$ and $-l \leq m \leq l .{ }^{5}$ The associated Legendre functions are denoted by $P_{l}^{m}$ and are defined for $l \geq 0$ in terms of

\footnotetext{
${ }^{5}$ Using standard trigonometric identities, one can show that $Y_{l}^{m}$ is a polynomial in $\Omega$ of degree $l$.
} 
Legendre polynomials: ${ }^{6}$

$$
P_{l}^{m}(x)=\left\{\begin{array}{ll}
(-1)^{m}\left(1-x^{2}\right)^{m / 2} \frac{\partial^{m} P_{l}(x)}{\partial x^{m}} & , m \geq 0 \\
(-1)^{m} \frac{(l-|m|) !}{(l+|m|) !} P_{l}^{|m|} & , m<0
\end{array} .\right.
$$

It is easy to show that if $F$ is a solution of (34), then the function $\hat{F}$ given by

$$
\hat{F}(x, y, \xi, \eta, \mu):=F(x, y, \xi, \eta,-\mu)
$$

is also a solution. From symmetry considerations, one can further show that moments with respect the $Y_{l}^{m}$ are identically zero when $l+m$ is odd. We therefore consider only moments for which $l+m$ is even.

For the quadrature set $Q$, we use Chebyshev-Legendre product quadrature set [36]. For a even integer $M$, points in the set have the form

$$
\Omega_{k_{1}, k_{2}}=(\xi, \eta, \mu)_{k_{1}, k_{2}}=\left(\mu_{k_{1}} \cos \phi_{k_{2}}, \mu_{k_{1}} \sin \phi_{k_{2}}, \mu_{k_{1}}\right)
$$

where $\mu_{k_{1}}, 1 \leq k \leq M / 2$, are the positive elements of the $M$-point, one-dimensional Gauss-Legendre quadrature set ${ }^{7}$ over the interval $[-1,1]$ and

$$
\phi_{k_{2}}=\frac{\pi}{2 M}\left(2 k_{2}+1\right), \quad k_{2}=1, \ldots, M .
$$

We choose this quadrature because it evaluates the polynomials in $\Omega$ up to degree $2 M-1$ exactly (up to roundoff). In particular, when the $P_{N}$ and $P P_{N}$ reconstructions agree - that is, when the $P_{N}$ reconstruction is positive on $Q$ - the half fluxes in the kinetic scheme (37), which involve polynomials degree $2 N+1$, are computed exactly (up to roundoff) whenever $M \geq 2 N$.

4.3. Properties of the Closure and Its Implementation. The current implementation of the $P P_{N}$ closure has two main benefits. First, it is local in space. Thus, even though the optimization algorithm (28) must be solved in each spatial cell at each successive time level, the solution procedure can be performed in parallel. Moreover, sharing of flux data between cells is only at the moment level, not at the kinetic level. For example, the fluxes in the one-dimensional scheme (32) are computed in the following way

$$
\begin{gathered}
\sum_{\mu_{k}>0} w_{k} \mu_{k} \mathbf{p}_{k} \frac{F_{i, k}^{n}-F_{i-1, k}^{n}}{\Delta x}=\frac{1}{\Delta x}\left\{\left(\sum_{\mu_{k}>0} w_{k} \mu_{k} \mathbf{p}_{k} F_{i, k}^{n}\right)-\left(\sum_{\mu_{k}>0} w_{k} \mu_{k} \mathbf{p}_{k} F_{i-1, k}^{n}\right)\right\} \\
\sum_{\mu_{k}<0} w_{k} \mu_{k} \mathbf{p}_{k} \frac{F_{i+1, k}^{n}-F_{i, k}^{n}}{\Delta x}=\frac{1}{\Delta x}\left\{\left(\sum_{\mu_{k}<0} w_{k} \mu_{k} \mathbf{p}_{k} F_{i+1, k}^{n}\right)-\left(\sum_{\mu_{k}<0} w_{k} \mu_{k} \mathbf{p}_{k} F_{i, k}^{n}\right)\right\},
\end{gathered}
$$

where the sums are calculated in each cell first before taking differences. (Note also that the products $w_{k} \mu_{k} \mathbf{p}_{k}$ are all precomputed.) The same strategy applies to implementations

\footnotetext{
${ }^{6}$ The spherical harmonics and associated Legendre functions have various normalizations and sign conventions. Our choice of constants is such that the harmonics form an orthonormal basis on $\mathbb{S}^{2}$. This choice has no effect on the results.

${ }^{7}$ Integration over negative values is determined by symmetry.
} 
in higher dimensions, such as (37). The fact that the algorithm requires relatively large numbers of local function evaluations, but a relatively small amount of data transfer means that it is well-suited to large scale implementation on massively parallel architectures, where memory transfer and latency are the bottlenecks to overall performance.

Another important benefit of the closure, which is not restricted to the numerical implementation given here, is the fact that it reduces to the standard $P_{N}$ closure whenever the reconstruction of the latter does not violate the positivity condition in (28). Thus, in our implementation, we can simply check whether or not the positivity condition is violated on the given quadrature set. If not, then we use the standard $P_{N}$ closure, for which the fluxes in (32) and (37) can be computed using the $P_{N}$ reconstruction (6). ${ }^{8}$ Typically the $P_{N}$ reconstruction will need to be corrected only around initial and boundary layers and regions of strong discontinuities.

The drawbacks to the current implementation are: (i) it is only first-order; and (ii) it is not asymptotic-preserving, in the sense of [16]. In particular it does not capture the diffusion limit of the transport equation [18]. This is the price to be paid for ensuring positivity of the underlying discrete solution to the kinetic equation. One could improve the formal accuracy to second order and still maintain positivity by using linear reconstructions with slope limiters for each quadrature angle in underlying kinetic discretizations (30) and (36). However, the use of limiters introduces nonlinearities and requires communication between spatial cells at the kinetic level, a complication we would like to avoid.

Efforts to develop a second-order AP scheme for the $P P_{N}$ system are currently underway, but no implementation exists at present. One idea is to use one of many possible AP schemes $[1,13,14,18,26]$ in regimes where the $P_{N}$ reconstruction is positive and then switch to the first-order scheme in regions where the $P_{N}$ reconstruction becomes negative, where the diffusion limit is not expected to be valid anyway.

\section{Numerical Results}

5.1. One-Dimension. We again consider the plane source problem from Example 1. Results for the particle concentration are plotted in Figures 5-7 for several values of $\mathrm{N}$ and at several times. For each $N$, the $P P_{N}$ solution is computed using a $2(N+1)$-point Gauss-Legendre quadrature set ${ }^{9}$ and are thus denoted $P P_{N^{-}} S_{2(N+1)}$. For comparison, a $P_{N}$ solution and a semi-analytic solution, taken from [10], is provided. In order to make a fair comparison, the $P_{N}$ solution is computed with the same kinetic scheme as the $P P_{N}$ solution. The only difference is that the $P P_{N}$ reconstruction is replaced by the usual $P_{N}$ ansatz (6). We make several observations about these results:

${ }^{8}$ This can be done efficiently by noting that the flux integrand in (3) can be written as

$$
\Omega \mathbf{p} F=\Omega \mathbf{p} \mathbf{p}^{T}\left\langle\mathbf{p}^{T} \mathbf{p}\right\rangle^{-1} \mathbf{u}
$$

Thus matrices needed to computed half-fluxes in the kinetic scheme are fixed integrals that can be precomputed. Only matrix-vector products with $\mathbf{u}$ need to be computed at run time. The necessary integrals can be done analytically or with the given quadrature rule associated to $\mathcal{Q}$.

${ }^{9}$ The $(N+1)$-point quadrature set corresponds to the standard $P_{N}$ solution. 
- The $P P_{N}$ closure propagates information from the initial condition faster than the $P_{N}$ closure.

- The wave behavior in the $P P_{N}$ closures is less pronounced than it is in the $P_{N}$ closure.

- The differences between $P_{N}$ and $P P_{N}$ decrease as $N$ and $t$ increase.

- The qualitative behavior of the $P P_{1}$ solution is similar to that of the maximum entropy solution in [4].

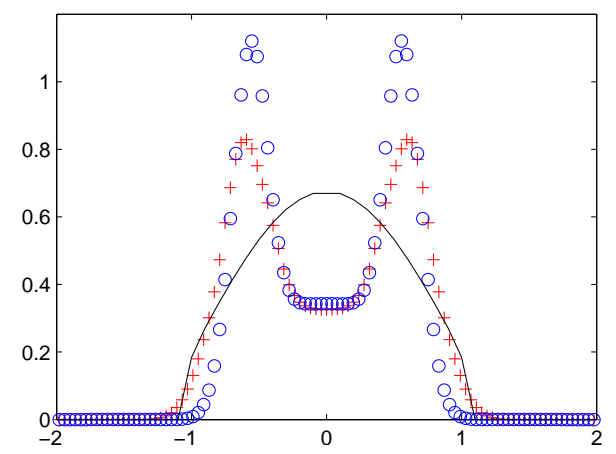

(a) $P_{1}-S_{4}$

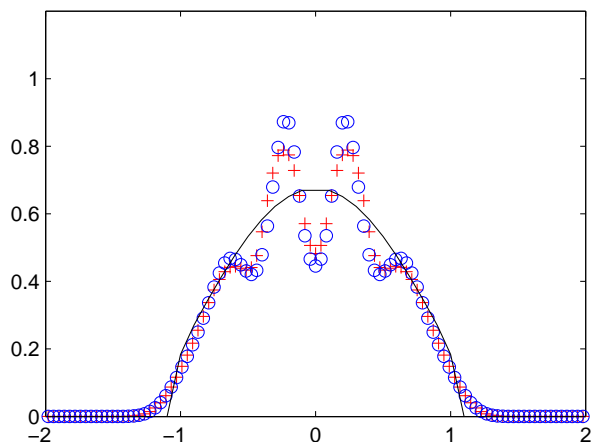

(c) $P_{5}-S_{12}$

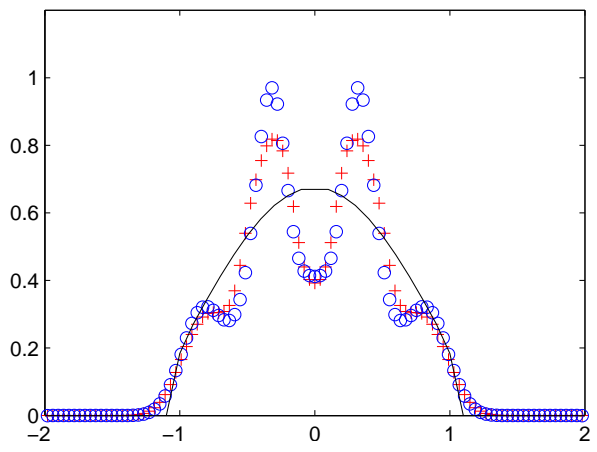

(b) $P_{3}-S_{8}$

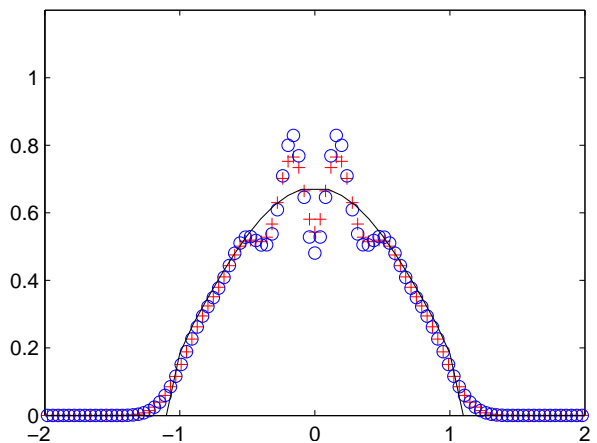

(d) $P_{7}-S_{16}$

FiguRE 5. Numerical results for the plane source at $t=1.0$. Solid Line: Semi-analytic solution; circles: $P_{N}$; crosses: $P P_{N}$. 


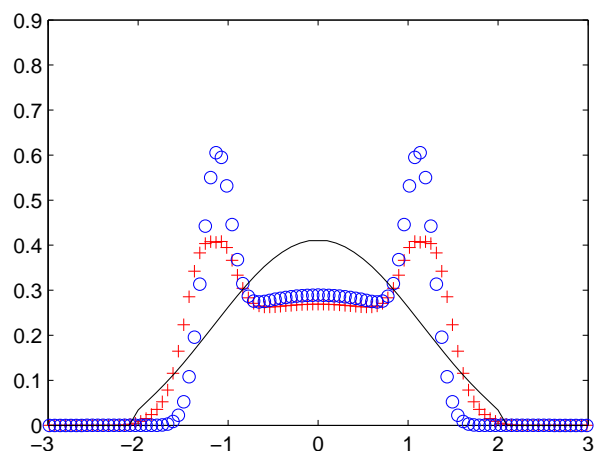

(a) $P_{1}-S_{4}$

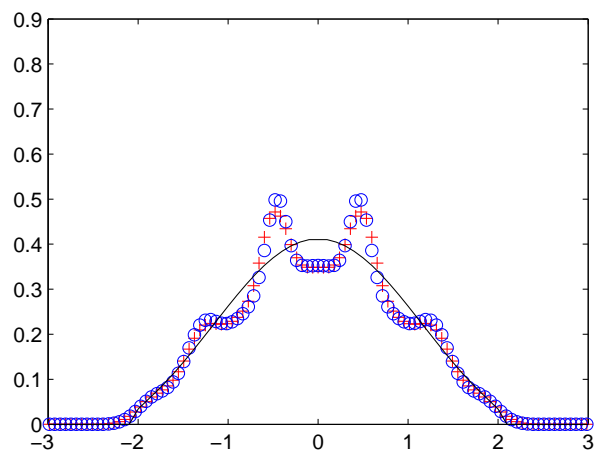

(c) $P_{5}-S_{12}$

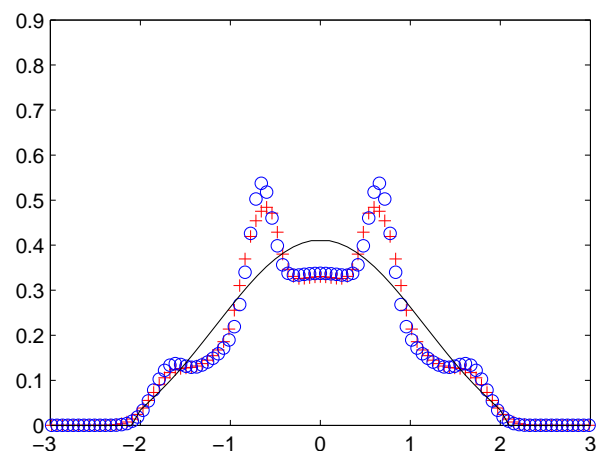

(b) $P_{3}-S_{8}$

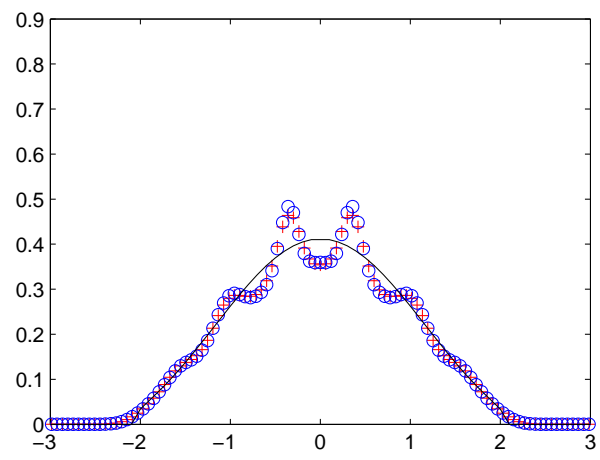

(d) $P_{7}-S_{16}$

FiguRE 6. Numerical results for the plane source at $t=2.0$. Solid Line: Semi-analytic solution; circles: $P_{N}$; crosses: $P P_{N}$. 


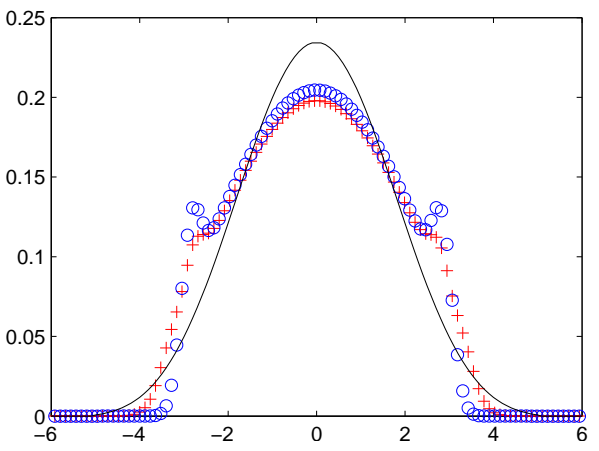

(a) $P_{1}-S_{4}$

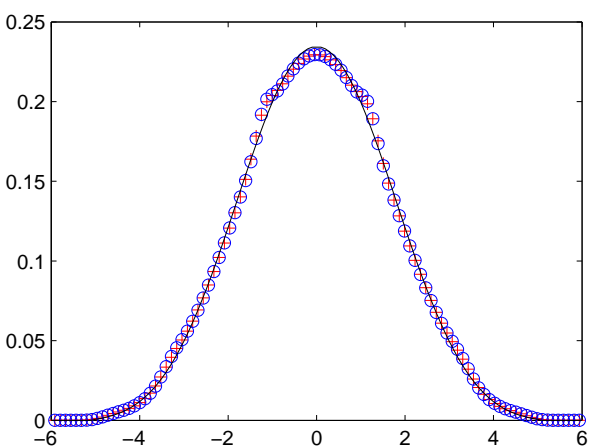

(c) $P_{5}-S_{12}$

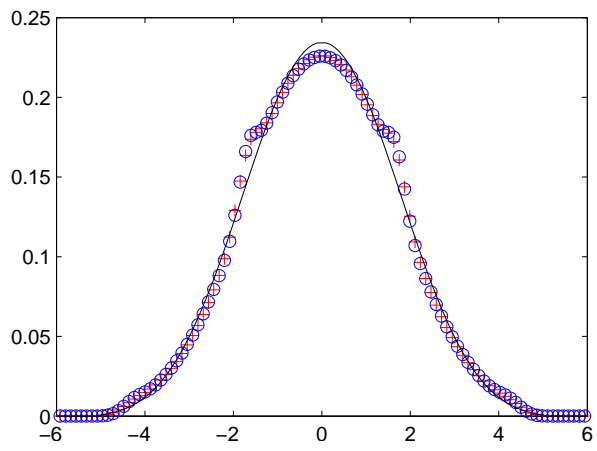

(b) $P_{3}-S_{8}$

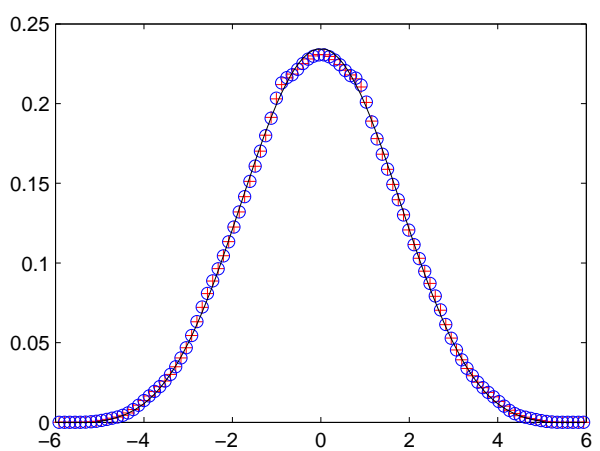

(d) $P_{7}-S_{16}$

Figure 7. Numerical results for the plane source at $t=5.0$. Solid Line: Semi-analytic solution; circles: $P_{N}$; crosses: $P P_{N}$.

5.2. Two Dimensions. We consider the pulsed line-source problem with $\sigma=1$ and initial particle concentration $\rho(x, y, 0)=\delta(x, y)+b$, where the background $b$ is a small, positive constant. All other moments intially zero. The reason for adding $b$ to the linesource is that quadprog sometimes fails to find a feasible solution to (27), due to (i) tolerances in the stopping criteria and in the constraints and also (ii) round-off error in the kinetic scheme itself. Adding the background is simply a quick fix to this problem. To ensure a robust algorithm, we make a conservative choice of $b=1.0^{-6}$, even though much smaller values often work. Future efforts will involve development of our own optimization algorithm which, among other things, will allow us to remove the background.

Plots of $\rho$ for a variety of simulations are given in Figures 8-13. In Figure 8, we compare the $P P_{N}$ quadrature with the underlying discrete ordinate solution. The only difference between the two calculations in that the discrete ordinate solution maintains values for 
each ordinate in memory and therefore does not need to reconstruct an ansatz. For a small quadrature set $(S 2)$, the $P P_{N}$ solution is quite similar to the discrete ordinate solution. In particular, rays effects are quite noticeable. However, for the larger $S_{8}$ quadrature set, the solutions are quite different.

Figure 9 compares $P P_{1}$ with the standard $P_{1}$ closure. As in the one-dimensional case, both closures are computed with the same kinetic scheme, but each with a different ansatz. Unlike the $P_{1}$ particle concentration, the $P P_{1}$ particle concentration remains positive at small times. In addition, the information from the pulse in the $P P_{1}$ solution moves faster than it does for the $P_{1}$ solution. However, at larger times, the two solutions are very similar. Figure 10 is a set of snapshots for the $P P_{1}-S_{8}$ closure.

Figures 11-13 repeat Figures 8-10, but with $N=3$. In these figures we note that there is a noticeable lack of rotational symmetry in the $P P_{N}$ solutions. Part of this phenomenon is due at least in part to the numerical grid. The $P_{3}$ solution shown in Figure 12 also has these artifacts at directions diagonal to the rectangular grid. Some part of the artifacts may be a ray effects from the underlying $S_{8}$ quadrature in the $P P_{3}$ solution. Nevertheless, the magnitude of these artifacts in the $P P_{3}$ solution is much smaller than the $S_{N}$ ray effects. 


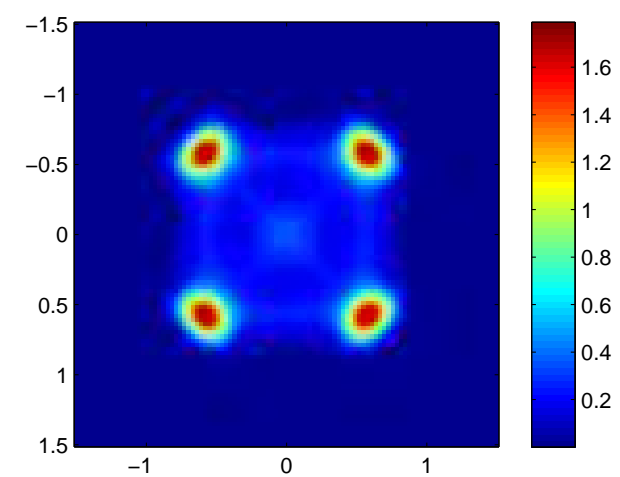

(a) $S_{2}$

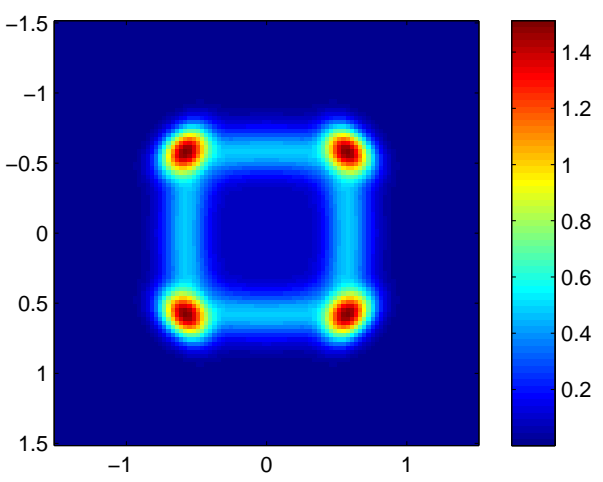

(c) $P P_{1}-S_{2}$

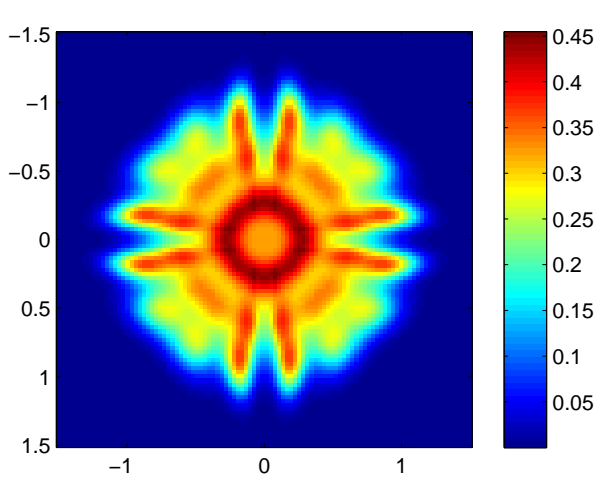

(b) $S_{8}$

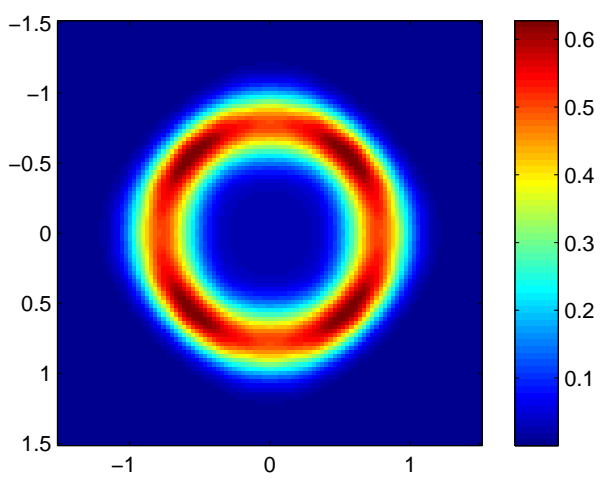

(d) $P P_{1}-S_{8}$

Figure 8. Comparison of $P P_{1}$ and the underlying discrete ordinate simulation $t=1.0$ for the line source problem. 


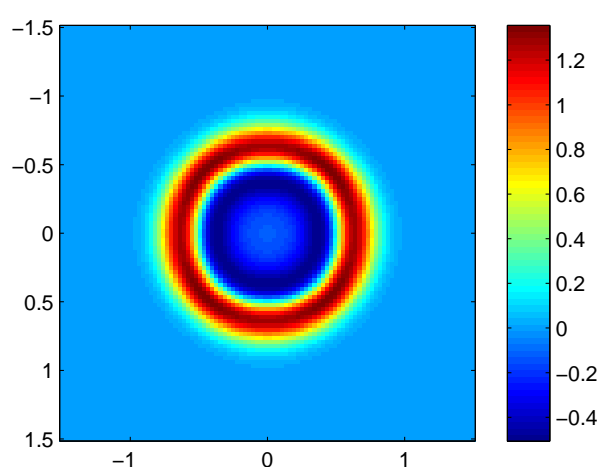

(a) $P_{1}, t=1.0$

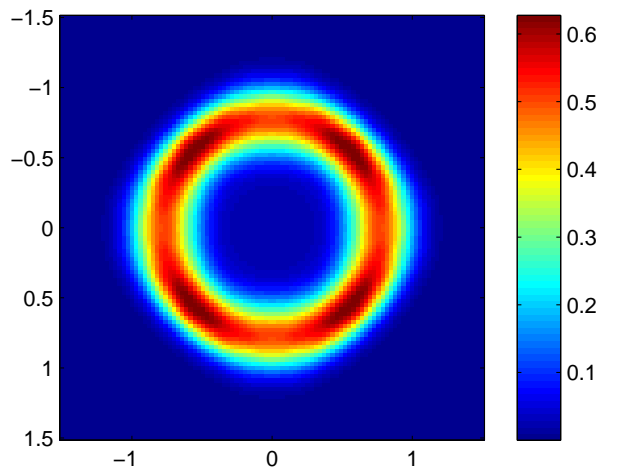

(c) $P P_{1}, t=1.0$

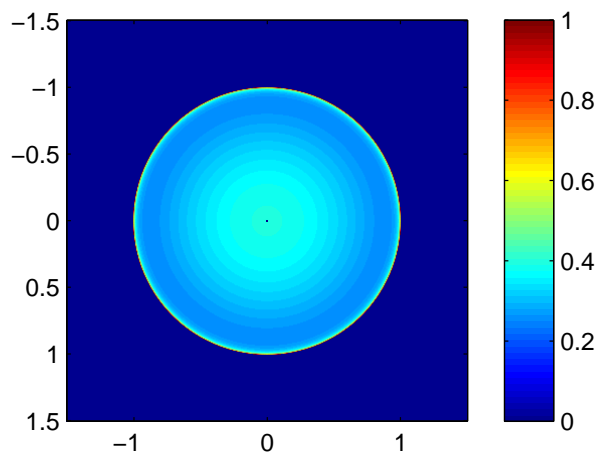

(e) Exact transport solution, $t=1.0$

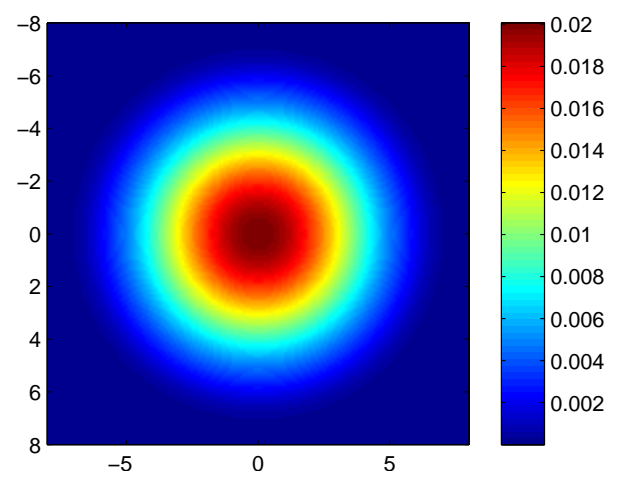

(b) $P_{1}, t=10.0$

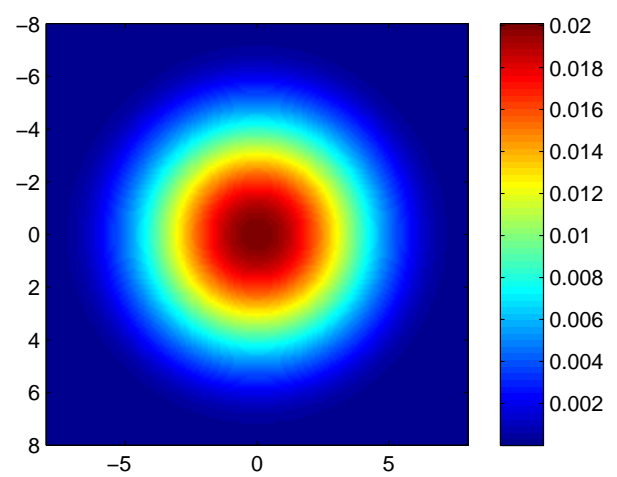

(d) $P P_{1}, t=10.0$

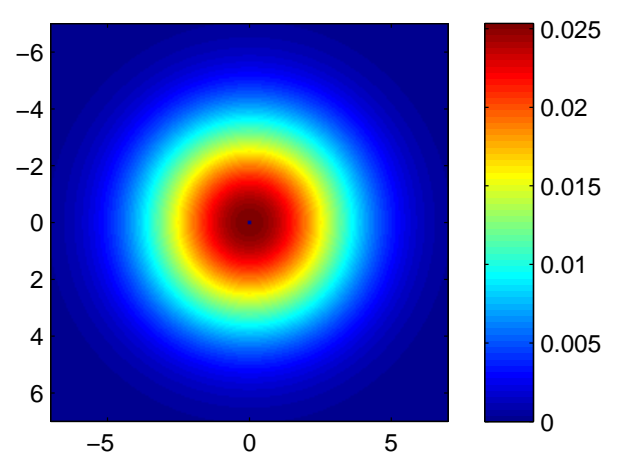

(f) Exact transport solution, $t=10.0$

Figure 9. Comparison of $P_{1}$ and $P P_{1}$ for the line source problem. 


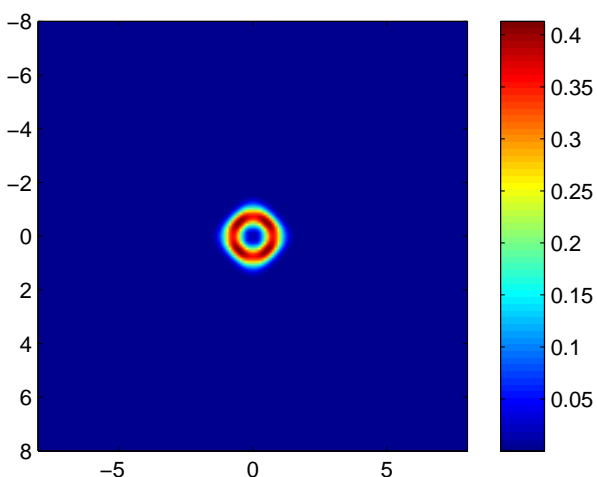

(a) $t=1.0$

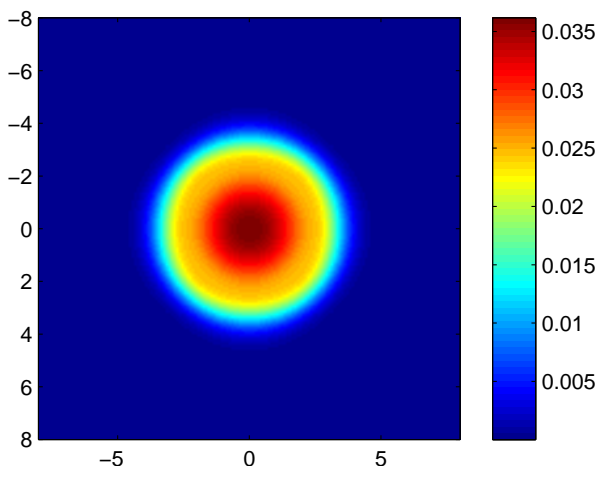

(c) $t=5.0$

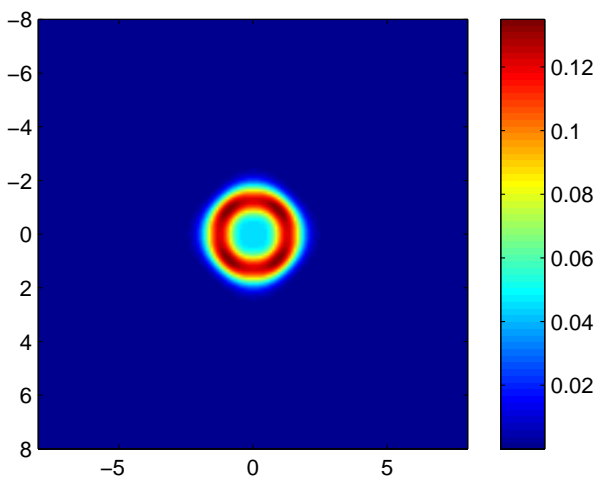

(b) $t=2.0$

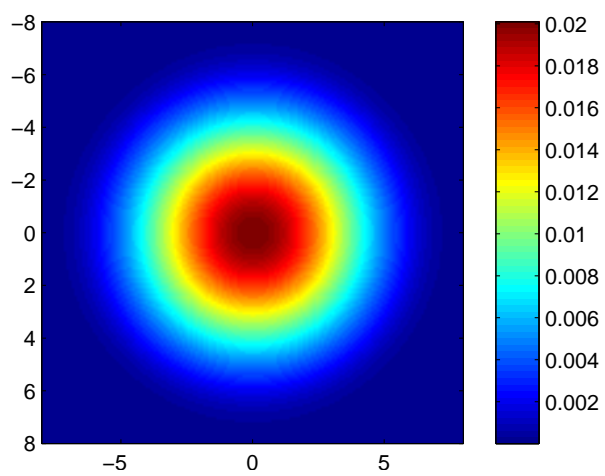

(d) $t=10.0$

Figure 10. Snapshots of $P P_{1}$ for the line source problem. 


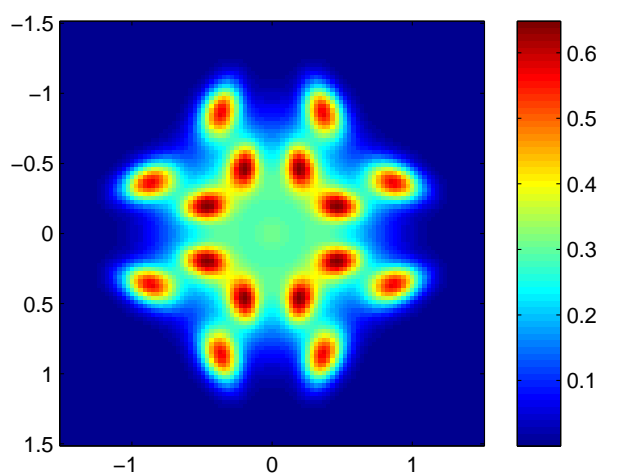

(a) $S_{4}$

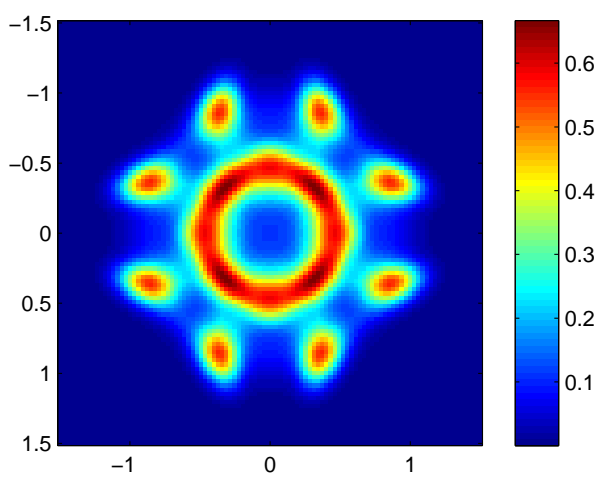

(c) $\mathrm{PP}_{3}-\mathrm{S}_{4}$

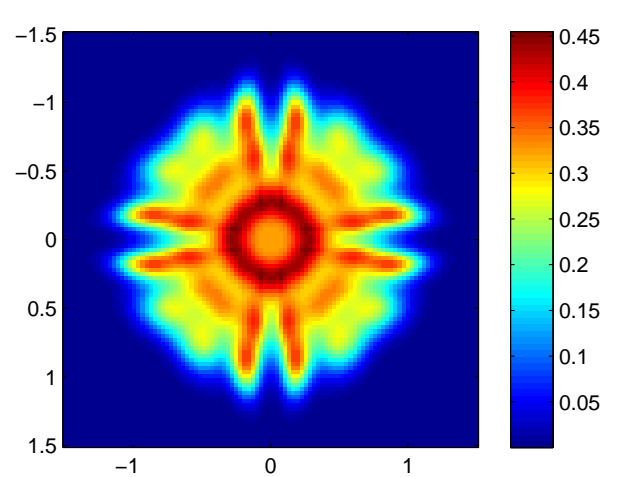

(b) $S_{8}$

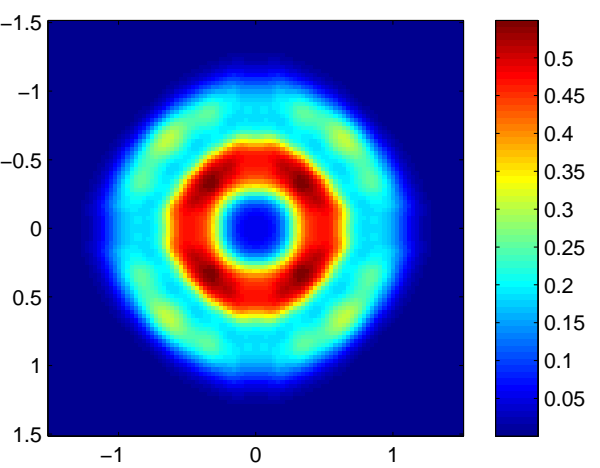

(d) $P P_{3}-S_{8}$

Figure 11. Comparison of $P P_{3}$ and the underlying discrete ordinate simulation $t=1.0$ for the line source problem. 


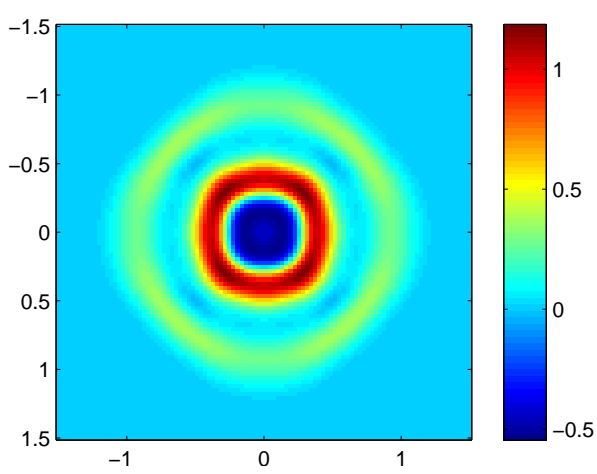

(a) $P_{3}, t=1.0$

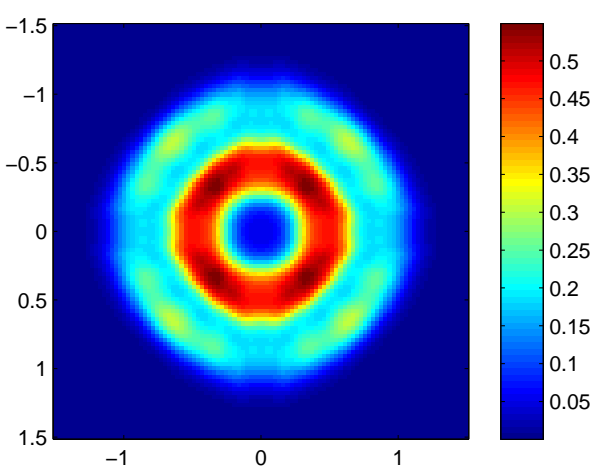

(c) $P P_{3}-S_{8}, t=1.0$

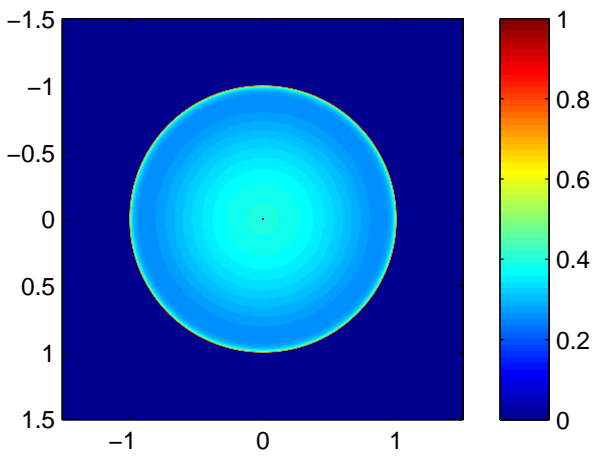

(e) Exact transport solution, $t=1.0$

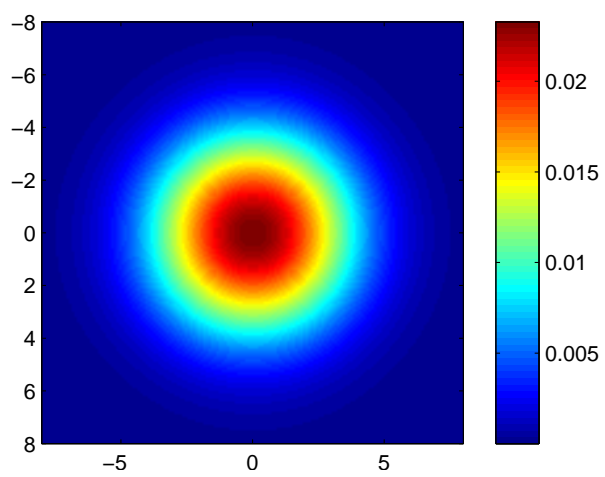

(b) $P_{3}, t=10.0$

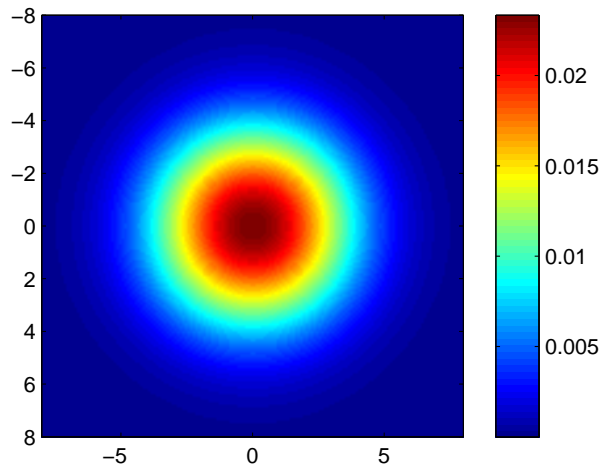

(d) $P P_{3}-S_{8}, t=10.0$

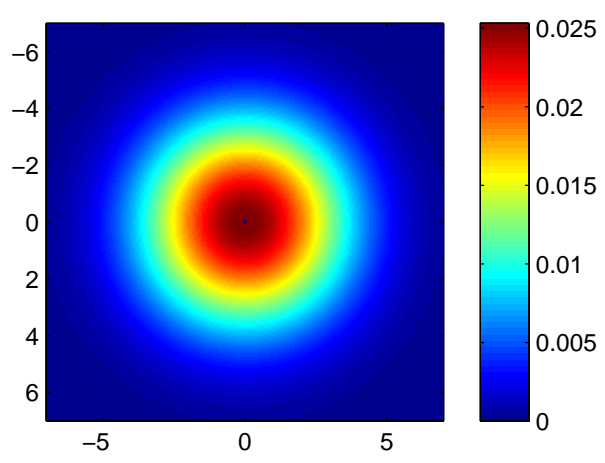

(f) Exact transport solution, $t=10.0$

Figure 12. Comparison of $P_{3}$ and $P P_{3}$ for the line source problem. 


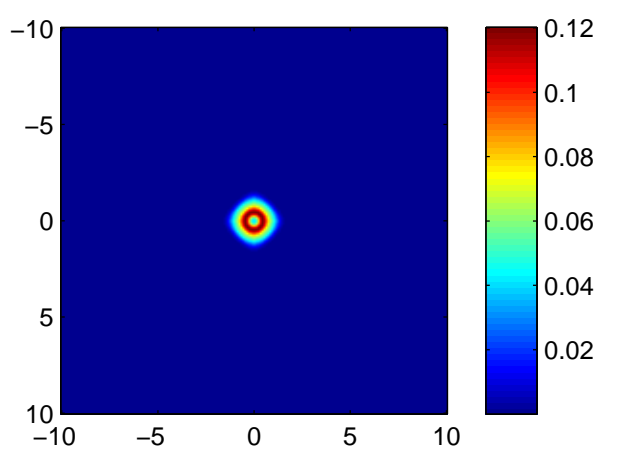

(a) $t=1.0$

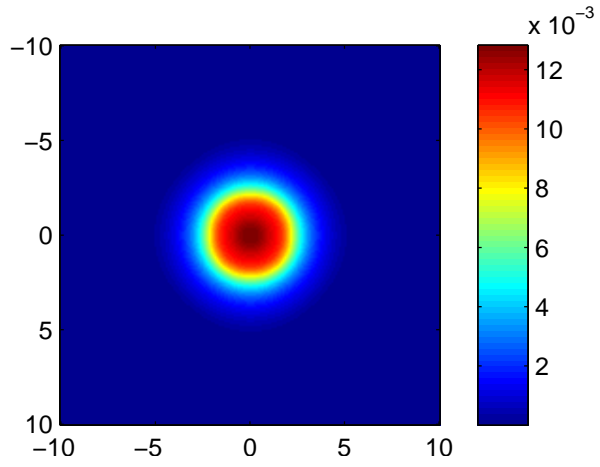

(c) $t=5.0$

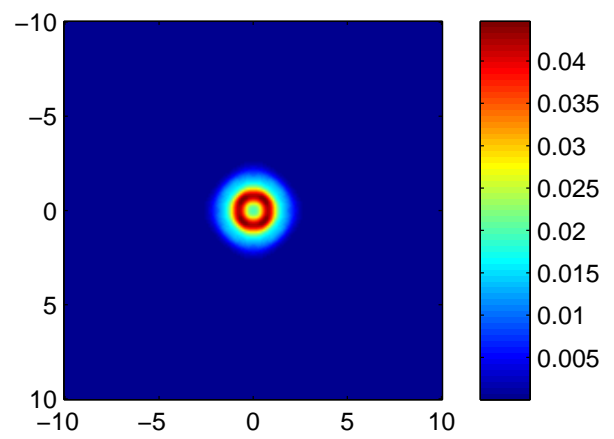

(b) $t=2.0$

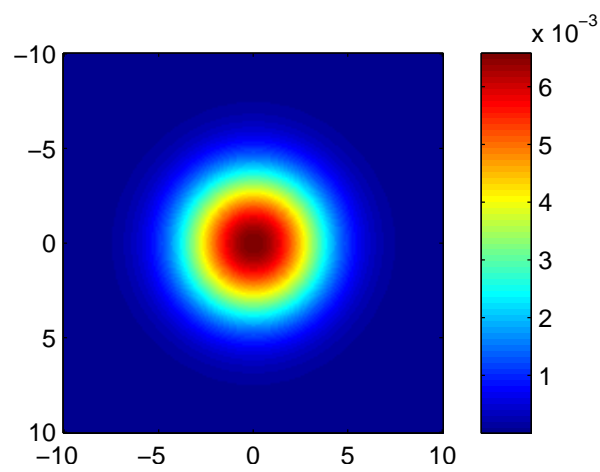

(d) $t=10.0$

Figure 13. Snapshots of $P P_{3}$ for the line source problem.

\section{Discussion and Conclusions}

We have motivated the development of a positive closure for the $P_{N}$ equations by showing that the kinetic reconstruction in the standard truncation closure can be negative, even in one-dimensional problems. We developed a closure for the $P_{N}$ equations, the $P P_{N}$ closure, that is based on the solution of a quadratic optimization problem. The new closure enforces positivity of the reconstruction at the points of a highly resolving quadrature set and reduces to the standard $P_{N}$ closure in regions where the standard reconstruction is positive.

In comparison with the standard $P_{N}$ truncation, our closure eliminates unphysical, negative particle concentrations while mitigating wave effects and slow particle speeds. Additionally, the $P P_{N}$ solutions are not plagued by ray effects found in discrete ordinates solutions. 
While we believe that the $P P_{N}$ method shows potential for solving large-scale transport problems, there is still a need for future work. Chiefly, the fact that our implementation is not asymptotic preserving needs to be addressed. Indeed, kinetic discretizations like (30) and (36) were long ago abandoned by the transport community for there lack of accuracy, especially in the diffusion limit. Implicit implementations are also needed for steady-state and stiff multi-physics problems. Finally, parallelization is required in order to make any implementation of the $P P_{N}$ system practical and computationally competitive.

\section{REFERENCES}

[1] M. L. Adams, Discontinuous finite element transport solutions in thick diffusive problems, Nucl. Sci. Eng., 137 (2001), pp. 298-333.

[2] G. B. Arfken And H. J. Weber, Mathematical Methods for Physicists, Academic Press, San Diego, 4th ed., 1995.

[3] T. A. Brunner, Forms of approximate radiation transport, Tech. Rep. SAND2002-1778, Sandia National Laboratories, 2002.

[4] T. A. Brunner And J. P. Holloway, One-dimensional Riemann solvers and the maximum entropy closure, J. Quant. Spec. Rad. Transfer, 69 (2001), pp. $543-566$.

[5] T. A. Brunner And J. P. Holloway, Two-dimensional time-dependent Riemann solvers for neutron transport, J. Comp Phys., 210 (2005), pp. 386-399.

[6] K. M. Case and P. F. Zweifel, Linear Transport Theory, Addison-Wesley, Reading, Mass., 1967.

[7] B. Davison, On the rate of convergence of the spherical harmonics method, Can. J. Phys., 38 (1960), pp. 1526-1545.

[8] J. Fleck and J. Cummings, An implicit Monte Carlo scheme for calculating time and frequency dependent nonlinear radiation transport, J. Comp. Phys., 8 (1971), pp. 313-342.

[9] B. D. GanAPOL, Solution of the one-group time-dependent neutron transport equation in an infinite medium by polynomial reconstruction, Nucl. Sci. Eng., 92 (1986), pp. 272-279.

[10] — Homogeneous infinite media time-dependent analytic benchmarks for X-TM transport methods development, tech. rep., Los Alamos National Laboratory, March 1999.

[11] B. D. Ganapol, C. T. Kelley, and G. C. Pomraning, Asymptotically exact boundary conditions for the $P_{N}$ equations, Nucl. Sci. Eng., 114 (1993), pp. 12-19.

[12] B. D. GANAPOL AND K. L. PEDDICORD, The generation of time-dependent neutron transport solutions in infinite media, Nucl. Sci. Eng., 64 (1977), pp. 317-331.

[13] C. D. Hauck And R. B. Lowrie, Temporal regularization of the $P_{N}$ equations, Multiscale Model. Simul., 7 (2008), pp. 1497-1524.

[14] C. D. Hauck, R. B. Lowrie, and R. M. McClarren, Numerical Methods For Balance Equations, Quaderni di Matematica, ch. "Methods for Diffusive Relaxation in the $P_{N}$ Equations". to appear.

[15] J. Jenal, P. J. Erickson, W. A. Rhodes, D. B. Simpson, and M. Williams, The generation of a computer library for discrete ordinates quadrature sets, Oak Ridge National Laboratory Report ORNL/TM-6023, 1977.

[16] S. JIN, Efficient asymptotic preserving (AP) schemes for some multiscale kinetic equations, SIAM J. Sci. Comp., 21 (1999), pp. 441-454.

[17] W. Kofink, Studies of the spherical harmonics method in neutron transport theory, Il Nuovo Cimento, 9 (1958), pp. 497-541.

[18] E. LARSEn, The asymptotic diffusion limit of discretized transport problems, Nucl. Sci. Eng., 112 (1992), pp. 336-346.

[19] E. W. LARsen And C. G. Pomraning, The $P_{N}$ theory as an asymptotic limit of transport theory in planar geometry -I: Analysis, Nucl. Sci. Eng, 109 (1991), pp. 49-75. 
[20] - The $P_{N}$ theory as an asymptotic limit of transport theory in planar geometry - II: Numerical results, Nucl. Sci. Eng., 109 (1991), pp. 76-85.

[21] C. D. Levermore, Boundary conditions for moment closures. Presented at Institute for Pure and Applied Mathematics University of California, Los Angeles, CA on May 27, 2009.

[22] E. E. Lewis AND W. F. Miller, Computational Methods of Neutron Transport, John Wiley and Sons, 1984.

[23] P. A. Markowich, C. A. Ringhofer, And C. Schmeiser, Semiconductor Equations, SpringerVerlag, New York, 1990.

[24] K. A. Mathews, On the propagation of rays in discrete ordinates, Nucl. Sci. Eng., 132 (1999), p. 155.

[25] R. G. McClarren, T. M. Evans, and R. B. Lowrie, Semi-implicit time integration for the $P_{n}$ equations, Trans. Am. Nuc. Soc., (2007).

[26] R. G. McClarren, T. M. Evans, R. B. Lowrie, and J. D. Densmore, Semi-implicit time integration for $P_{N}$ thermal radiative transfer, J. Comp. Phys., 227 (2008), pp. 7561-7586.

[27] R. G. McClarren, J. P. Holloway, and T. A. Brunner, Analytic $P_{1}$ solutions for time-dependent, thermal radiative transfer in several geometries, J. Quant. Spec. Rad. Transfer, 109 (2008), pp. 389-403.

[28] R. G. McClarren, J. P. Holloway, and T. A. Brunner, On solutions to the $P_{n}$ equations for thermal radiative transfer, J. Comput. Phys., 227 (2008), pp. 2864-2885.

[29] A. Mezzachppa And O. E. B. Messer, Neutrino transport in core collapse supernovae, J. Comput. Appl. Math., 109 (1999), pp. 281-319.

[30] D. Mihalas and B. W. Mihalas, Foundations of Radiation Hydrodynamics, Dover, New York, 1999.

[31] R. D. O'Dell And R. E. Alcouffe, Transport calculations for nuclear analyses: Theory and guidelines for effective use of transport codes, Los Alamos Scientific Laboratory Report LA-10983-MS, 1987.

[32] G. L. Olson, L. H. Auer, And M. L. Hall, Diffusion, $P_{1}$, and other approximate forms of radiation transport, J. Quant. Spec. Rad. Transfer, 64 (2000), pp. 619-634.

[33] G. C. Pomraning, Variational boundary conditions for the spherical harmonics approximation to the neutron transport equation, Ann. Phys., 27 (1964), pp. 193-215.

[34] G. C. Pomraning, Radiation Hydrodynamics, Pergamon Press, New York, 1973.

[35] I. V. Serov, T. M. John, And J. E. Hoogenboom, A new effective Monte Carlo Midway coupling method in MCNP applied to a well logging problem, Appl. Rad. and Isotopes, 49 (1998), pp. 1737-1744.

[36] W. F. WALters, Use of the Chebyshev-Legendre quadrature set in discrete-ordinate codes, Los Alamos National Laboratory Report LA-UR-87-3621, 1987.

[37] G. YAun, Studies of the spherical harmonics method in neutron transport theory, J Math. Phys., 41 (2000), pp. 867-874. 